\title{
Design of Howland current sources using differential evolution optimization
}

\author{
Kaue Felipe Morcelles ${ }^{1,4}$, Lucas Hermann Negri ${ }^{2}$, Pedro Bertemes-Filho ${ }^{3}$ \\ 1. State University of Santa Catarina. Joinville. Brazil \\ 2. Federal Institute of Education, Science and Technology of Mato Grosso do Sul. Nova Andradina, Brazil. \\ 3. State University of Santa Catarina. Joinville, Brazil \\ 4. E-mail any correspondence to: kaue.morcelles@edu.udesc.br
}

\begin{abstract}
Howland circuits have been widely used in Electrical Bioimpedance Spectroscopy applications as reliable current sources. This paper presents an algorithm based on Differential Evolution for the automated design of Enhanced Howland Sources according to arbitrary design constraints while respecting the Howland ratio condition. Results showed that the algorithm can obtain solutions to commonly sought objectives, such as maximizing the output impedance at a given frequency, making it a versatile method to be employed in the design of sources with specific requirements. The mathematical modeling of the source output impedance and transconductance, considering a non-ideal operational amplifier, was validated against SPICE simulations, with results matching up to $10 \mathrm{MHz}$.
\end{abstract}

Keywords: bioimpedance, differential evolution, Howland source, optimization

\section{Introduction}

Electrical Bioimpedance Spectroscopy (EBS) has been widely used over the last 50 years for biomedical applications [1], such as cancer diagnoses [2], monitoring of physiological activity (lung fluids [3], muscle contractions [4] and cardiac output [5]) and real-time tomography [6]. EBS uses the correlation between material constitution/geometry and electrical impedance to characterize the tissue [7].

The Enhanced Howland Source (EHS) is a current pump used in EBS measurements consisting of one operational amplifier and five resistors [8]. Its parameters (transconductance, output impedance, voltage compliance, noise) can be designed by selecting adequate resistor values and OPAMP model $[9,8,7]$. The transconductance value and minimum voltage compliance are usually fixed by the application requirements, whereas the output impedance and noise should be maximized and minimized, respectively, to avoid measurement errors. Especially concerning the output impedance (Zout), the EHS has limitations in high frequencies.

This paper proposes the usage of differential evolution to design a Howland current source with optimal output impedance according to arbitrary project constraints.

\section{Methodology}

The Differential Evolution (DE) optimization method [10] was employed to select the optimal resistor values $\left(R_{1}\right.$, $R_{2 A}, R_{2 B}, R_{3}$, and $R_{4}$ ) for the Howland Source, according to the design goals (see Figure 2).

Being a black-box, population-based metaheuristic, the DE does not use gradient information to guide the optimization process. Instead, DE relies in a fitness function, that determines the quality of a candidate solution. Specifically, the JADE variant of DE was employed [11] to make the optimization process more robust, as JADE automatically updates the DE control parameters. Figure 1 shows a flowchart that describes the general steps of the optimization method.

The optimization procedure is based on the mathematical modeling of the Howland Source, and was performed to maximize the output impedance at the highest frequency of operation. A total of 50 candidate solutions were evolved during 1000 iterations of the optimization procedure.

The mathematical model of the Howland Source used in this work includes the resistor set $\left(R_{1}, R_{2 A}, R_{2 B}, R_{3}, R_{4}\right)$ and the Operational Amplifier (OPAMP) non-idealities that potentially affects the parameters of interest, namely the finite open-loop gain $A$, finite cut-off frequency $f_{C}$, 
common mode input resistance $R_{C M}$. As the algorithm can yield large values of resistors, the input capacitance of the OPAMP $\left(C_{i n}\right)$ must also be included on the model, in order to predict instability and high frequency peaks due to phase margin reduction at the negative feedback loop.
The output impedance and transconductance were calculated using Equations 1 and 2. Maximum load capability was evaluated using the model presented by a previous work [12]. The equations were derived from the Howland circuit shown in Figure 2.

$$
\begin{aligned}
& Z_{\text {out }}= \frac{R_{2 A}\left(s+\frac{1}{2 C_{i n}\left(R_{C M}\left\|R_{1}\right\| R_{2 A}\right)}\right)}{A\left(s+\frac{1}{2 C_{i n}\left(R_{C M}\left\|R_{3}\right\| R_{4}\right)}\right)}+\left(\frac{R_{2 A}}{R_{2 B}}+1\right)\left(s+\frac{1}{2 C_{i n}\left(R_{C M}\left\|R_{1}\right\| R_{2 A}\right)}\right)-\frac{1}{2 C_{i n} R_{2 A}} \\
&-\frac{A}{R_{2 B}\left(\left(2 C_{i n} s+\frac{1}{\left(R_{C M}\left\|R_{3}\right\| R_{4}\right)}\right)\left(\frac{s}{f_{C} \pi}+1\right)+\frac{A}{R_{4}}\right)} \\
& G=\frac{A R_{4} R_{C M}}{\left(\frac{s}{2 f_{c} \pi}+1\right)\left(R_{C M}\left(R_{2 B} R_{3}\left(\frac{A}{\frac{s}{2 f_{C} \pi}+1}+1\right)+R_{2 B} R_{4}\right)+C_{i n} R_{2 B} R_{3} R_{4} R_{C M} s+R_{2 B} R_{3} R_{4}\right)}
\end{aligned}
$$

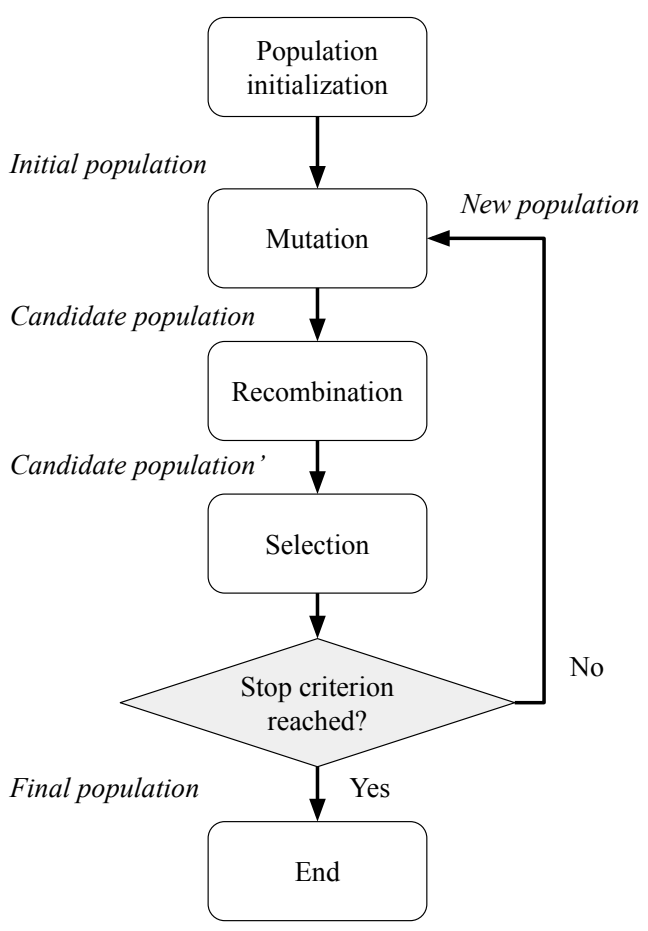

Figure 1: Flowchart that describes the main steps of the optimization method.

The fitness function employed in the selection step of the JADE method is given by equation 3, where $p_{1}, p_{2}, p_{3}$, and $p_{4}$ (Equations 4, 5, 6, 7) are penalty factors that implements the design constraints, $\alpha$ is the penalization weight that is calibrated (increased until all design constraints are met), $r l_{\max }$ is given by equation 8, and $G_{\text {sample }}$ is a vector with samples of the transconductance magnitude in the $100 \mathrm{kHz}$ to $1 \mathrm{MHz}$ band. For the Optimized Case 1, the transconductance variance was not taken into account, therefore $\beta=0$ at

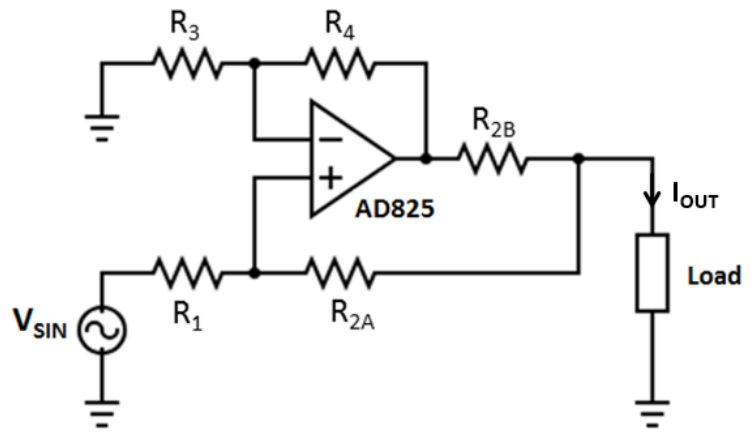

Figure 2: Schematic circuit of the ground loaded modified Howland Current Source.

equation 6. For the Optimized Case 2 , the value $\beta=10^{-9}$ was used instead.

$$
\begin{array}{r}
\text { fitness }=Z_{\text {out }}-\alpha\left(p_{1}+p_{2}+p_{3}+p_{4}\right) \\
p_{1}=\left|\frac{R_{4}}{R_{3} R_{2 b} G_{p}}-1\right| \\
p_{2}=\left|\frac{\left(R_{2 a}+R_{2 b}\right) R_{3}}{R_{1} R_{4}}-1\right| \\
p_{3}=\beta \frac{\operatorname{var}\left(G_{\text {sample }}\right)}{\text { median }\left(G_{\text {sample }}\right)} \\
p_{4}=1-\frac{r l_{\text {max }}}{R l_{\text {max }}} \\
r l_{\text {max }}=\frac{V_{\text {sat }}}{V_{\text {in }} G p}\left(1+\frac{R_{2 B}}{R_{2 A}+R_{1}}\right)^{-1}
\end{array}
$$

Ethical approval

The conducted research is not related to either human or animal use. 


\section{Results}

In order to validate the algorithm, a case study was performed, consisting of the design of a Howland circuit with a set of pre-defined requirements (Table 1 ). The operational amplifier model used was the AD825, from Analog Devices ${ }^{\mathrm{TM}}$. The algorithm was configured to automatically design the resistor values of the Howland Source, maximizing the output impedance modulus at the maximum desired frequency (in this case $1.2 \mathrm{MHz}$ ), while respecting the design requirements and the Howland ratio condition [9]. Also, the resistors were constrained to values between 0.05 and $100 \mathrm{k} \Omega$. The first round of optimization evaluated a solution (Optimized Case 1 ) consisting of $R_{1}=4.303990 \mathrm{k} \Omega, R_{2 A}=0.678260 \mathrm{k} \Omega$, $R_{2 B}=2.230320 \mathrm{k} \Omega, R_{3}=7.478330 \mathrm{k} \Omega$ and $R_{4}=5.053760$ $k \Omega$. The resulted impedance and transconductance curves are compared with two cases: Case 1 consisted of the common approach of making $R_{2 B}=R_{2 A}=R_{3}=R_{4}=$ $R_{1} / 2$ and $G=1 / R_{2 B}$, a combination that is not yet optimized for output impedance [12]. In this case, the requirements of Table 1 were satisfied using $R_{2 B}=3.3 \mathrm{k} \Omega$ [12]. Case 2 verified the impact of increasing the feedback resistors, making $G=1 / R_{2 B}, R_{2 A}=R_{3}=R_{4}=100 R_{2 B}$ and $R_{1}=R_{2 A}+R_{2 B}$. This approach reduces the feedback current, increasing voltage swing. In this case, $R_{2 B}=3.3$ $k \Omega$ was also used. The resulted output impedance and transconductance (no load) are shown in Figures 3 and 4.

Table 1: Electrical parameters required for designing the Howland current source.

\begin{tabular}{|lr|}
\hline \hline Transconductance $(G p)$ & $303.03 \mu \mathrm{S}$ \\
Frequency Range & $10 \mathrm{~Hz}-1.2 \mathrm{MHz}$ \\
Maximum Load $\left(R l_{\text {max }}\right)$ & $2.0 \mathrm{k} \Omega$ \\
Supply Voltage $\left(V_{\text {sat }}\right)$ & $\pm 5.0 \mathrm{~V}$ \\
Input Voltage $\left(V_{\text {in }}\right)$ & $1.65 \mathrm{~V}$ \\
Operational Amplifier & $\mathrm{AD} 825$ \\
\hline \hline
\end{tabular}

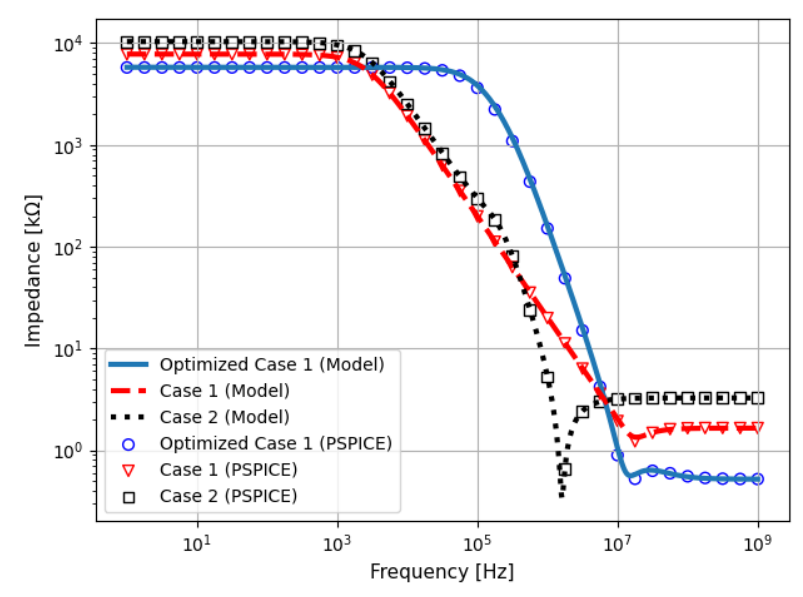

Figure 3: Output impedance of the optimized and nonoptimized Howland Source circuits.

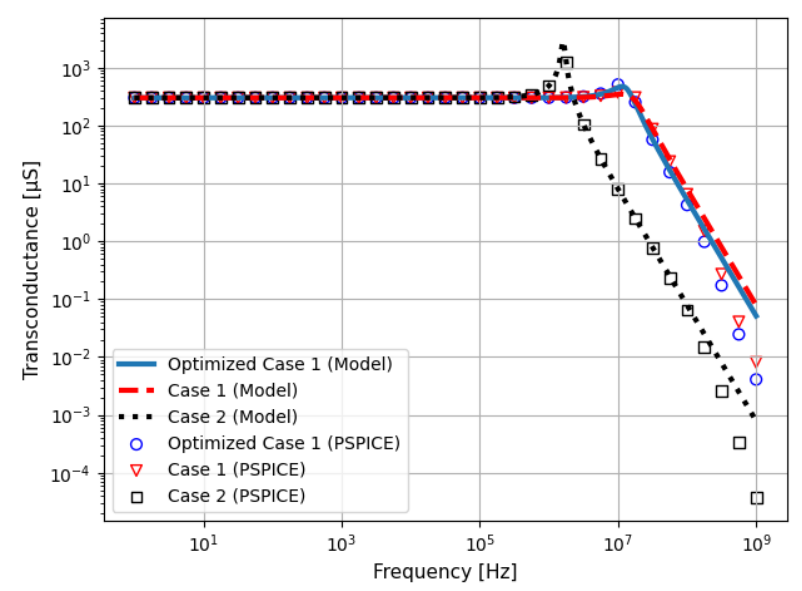

Figure 4: Transconductance curve of the optimized and non-optimized Howland Source Circuit.

At maximum frequency $(1.2 \mathrm{MHz})$, Case 1 and Case 2 obtained $16.80 \mathrm{k} \Omega$ and $2.85 \mathrm{k} \Omega$ respectively, whereas the Optimized Case 1 obtained $107.61 \mathrm{k} \Omega$, which is 6.4 times higher than Case 1 . In contrast, increasing the feedback resistors (Case 2) incremented the output impedance only at low frequencies, leading to a reduction in $Z_{\text {out }}$ at high frequencies due to interactions with the input capacitance of the operational amplifier. All cases presented transconductance peaking at high frequencies, but due to the strong effects of the input capacitance Case 2 presented the largest current error. Finally, it can be seen that the curves generated with PSPICE and the ones evaluated using the equations here presented agree up to $10 \mathrm{MHz}$.

To verify the dependence of the output current on the load, Figure 5 shows the transconductance of the Optimized Case 1 curves for three different loads. It can be seen that the high frequency current peaking increases with the load, which is the opposite of what happens in Case 1 . To verify the time domain effects of peaking, Figure 6 shows the wave-shape of the output current of Case 1 and the Optimized Case 1, considering three different loads. The Optimized Case 1 presented a higher overshoot value that increased with the load, whereas Case 1 obtained lower overshoot that decreased with the load. This behavior may be problematic in sensitive applications measuring high loads, as high overshoot degrades signal performance and resolution, especially when using square waves or other discrete signals. Peaking can be reduced by placing a capacitor in parallel with $R_{4}$, but this has the cost of significantly reducing the output impedance at high frequencies.

To minimize the current peaking, another run of optimization was performed, generating the resistor set called Optimized Case 2, using $\beta=10^{-9}$ at equation 6. This approach maximizes the output impedance while lowering the amount of peaking. The algorithm evaluated the solution: $R_{1}=4.187160 \mathrm{k} \Omega, R_{2 A}=1.192640 \mathrm{k} \Omega$, 


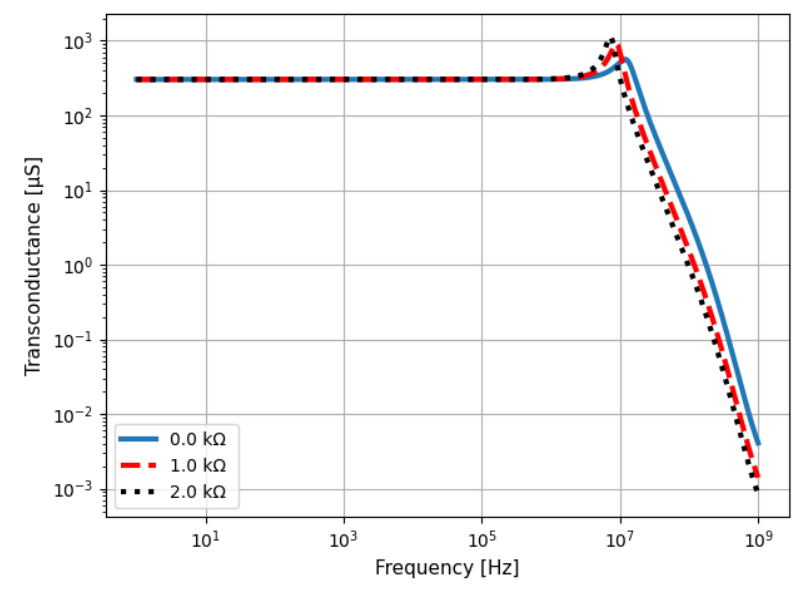

(a)

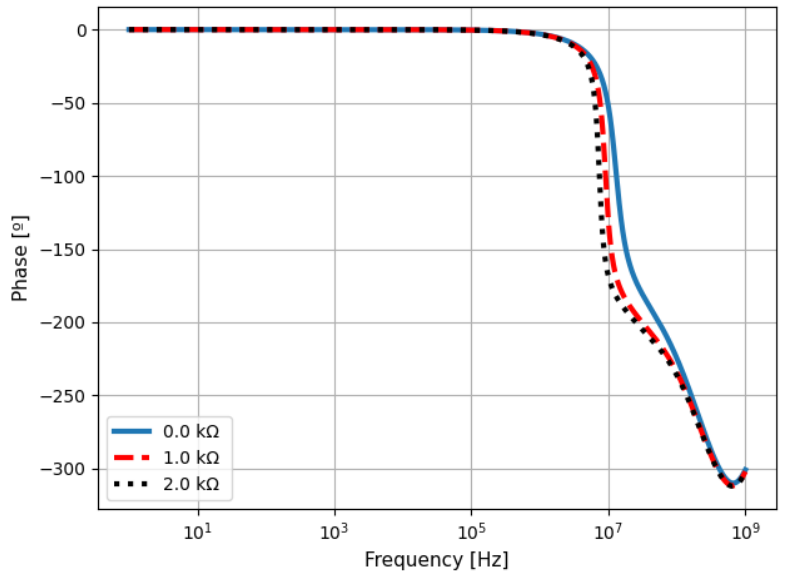

(b)

Figure 5: Transconductance (a) magnitude and (b) phase curves of the Optimized Case 1 for three different loads.

$R_{2 B}=4.438410 \mathrm{k} \Omega, R_{3}=2.529140 \mathrm{k} \Omega$ and $R_{4}=3.401290$ $k \Omega$. The curve had a peak of $6.20 \%$ (in contrast with Optimized Case 1, with peak of $57.22 \%$ ). However, the $Z_{\text {out }}$ decreased to the level of Case 1 , being slightly higher $(20 \mathrm{k} \Omega)$ at maximum frequency. This result illustrates a trade-off between output impedance and high frequency peaking presented in the circuits generated by the algorithm, and the designer should decide which parameter is more important for the application.

Although the Optimized Case 1 presented higher current peaking than the Case 1 and the Optimized Case 2 , the errors within the bandwidth of interest (up to 1.2 $\mathrm{MHz}$ ) were significantly smaller, as shown in Tables 2 and 3. In the Optimized Case 1, both magnitude and phase errors were smaller even at maximum load $(2.0 \mathrm{k} \Omega$ ), which is the case of maximum peaking for the Optimized Case 1. This result was expected, as higher output impedance typically results in lower output current variations with the load.

Although the JADE algorithm is capable of finding the resistor combination that yields maximum output impedance, it does not take into account the variations

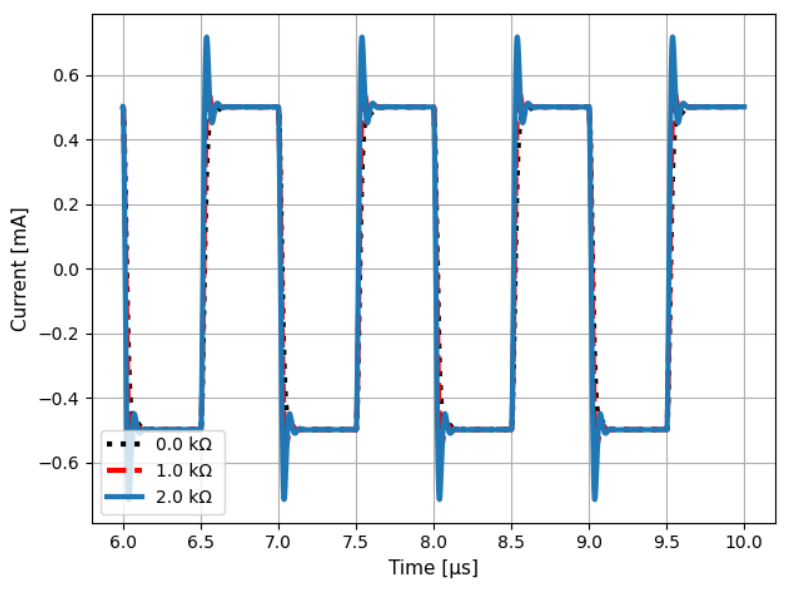

(a)

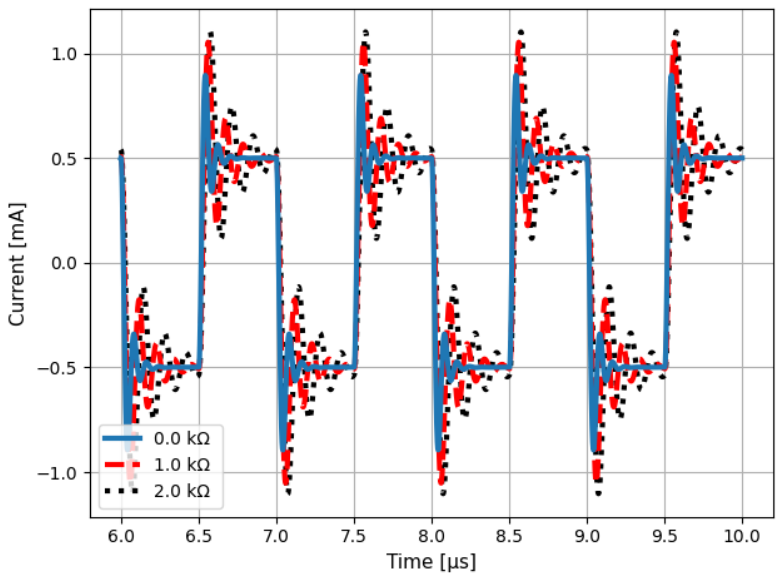

(b)

Figure 6: Output current of the (a) Case 1 and (b) Optimized Case 1 circuits for three different loads and $1 \mathrm{MHz}$ square wave input voltage.

Table 2: Output Current Error (Magnitude and Phase) for different Loads (at $100 \mathrm{kHz}$ ).

\begin{tabular}{cccc}
\hline \hline Case & Load $[k \Omega]$ & Magnitude $[\%]$ & Phase $\left[{ }^{\circ}\right]$ \\
\hline \multirow{3}{*}{ Case 1 } & 0.0 & 0.62 & -0.36 \\
& 1.0 & 1.13 & -0.65 \\
& 2.0 & 1.63 & -0.93 \\
\hline \multirow{3}{*}{ Optimized 1 } & 0.0 & 0.52 & -0.30 \\
& 1.0 & 0.55 & -0.31 \\
& 2.0 & 0.57 & -0.33 \\
\hline \multirow{2}{*}{ Optimized 2 } & 0.0 & 0.56 & -0.32 \\
& 1.0 & 0.99 & -0.57 \\
& 2.0 & 1.42 & -0.81 \\
\hline \hline
\end{tabular}

due to fabrication tolerances. Therefore, Monte Carlo simulations were performed in PSPICE to evaluate the robustness of the circuit solution. In this test, precision resistors with $0.05 \%$ tolerances were considered. The Monte Carlo simulation ran 10000 cases (using Gaussian distribution), checking the variation of the output impedance value at the maximum frequency (1.2 
Table 3: Output Current Error (Magnitude and Phase) for different Loads (at $1.2 \mathrm{MHz}$ ).

\begin{tabular}{cccc}
\hline \hline Case & Load $[k \Omega]$ & Magnitude [\%] & Phase $\left[{ }^{\circ}\right]$ \\
\hline \multirow{3}{*}{ Case 1} & 0.0 & 7.55 & -4.32 \\
& 1.0 & 13.50 & -7.75 \\
& 2.0 & 19.33 & -11.14 \\
\hline \multirow{3}{*}{ Optimized 1 } & 0.0 & 6.39 & -3.67 \\
& 1.0 & 6.91 & -3.82 \\
& 2.0 & 7.53 & -4.01 \\
\hline \multirow{3}{*}{ Optimized 2 } & 0.0 & 6.78 & -3.87 \\
& 1.0 & 11.95 & -6.84 \\
& 2.0 & 17.10 & -9.81 \\
\hline \hline
\end{tabular}

$\mathrm{MHz})$. Results are shown in Table 4, with the mean $(\mu)$, standard deviation $(\sigma)$ and coefficient of variation $(\mathrm{CV})$ of the output impedance of each case. It can be seen from Table 4 that the solutions provided by the JADE algorithm are less robust than Case 1, but still provide a significant increase in output impedance at the desired frequency. Better robustness can be obtained using resistors with lower tolerances.

Table 4: Monte Carlo simulation results (at 1.2 $\mathrm{MHz}$ ), showing the mean output impedance $(\mu)$, standard deviation $(\sigma)$ and the coefficient of variation (CV) of each case over 10000 runs using $0.05 \%$ tolerance resistors.

\begin{tabular}{cccc}
\hline \hline Case & $\mu[k \Omega]$ & $\sigma[k \Omega]$ & $\mathrm{CV}[\%]$ \\
\hline Case 1 & 16.26 & 1.18 & 7.25 \\
\hline Optimized & 103.41 & 29.89 & 28.90 \\
\hline Optimized 2 & 18.67 & 2.05 & 10.96 \\
\hline \hline
\end{tabular}

\section{Conclusion}

The paper investigated the use of differential evolution for designing and modelling a Howland current source with optimum output impedance and complying with project constraints. It was focused on the output impedance optimization at $1.2 \mathrm{MHz}$, but can be extrapolated to different frequencies and parameters. It was found a trade off between the high frequency transconductance error and output impedance during the optimization process. Decreasing the transconductance peak can be done by minimizing transconductance variance, however at price of having a decrease in the output impedance. Nevertheless, it was showed that the mathematical model and simulations agreed significantly up to $10 \mathrm{MHz}$. The analytical nature of the models make them more suitable for iterative optimization algorithms, providing less computational cost and time when compared with numerical and SPICE based optimization approaches. This is a promising output to be used in the design of bioimpedance measurement circuits which, in turns, are very dependent on the clinical application and the biological samples under study. Future work will focus on extracting experimental data, to verify how the algorithm complies with parasitic stray capacitances, non-idealities of the components and the limited availability of discrete valued resistors.

\section{Conflict of interest \\ Authors state no conflict of interest.}

\section{References}

1. Grimnes S and Martinsen OG. Bioimpedance and Bioelectricity basics. $3^{\text {rd }}$ ed. Academic press, 2015

2. Svoboda RM, Gharia MJ, Shell J and Gregory WD. Bioimpedance measurement as an assessment of margin positivity in Mohs surgical specimens of nonmelanoma skin cancer: Management implications. Journal of the American Academy of Dermatology 2018; 79:591-3. DOI: 10.1016/j.jaad.2018.02.075

3. Zellner JL, Spinale FG and Crawford FA. Bioimpedance: A novel method for the determination of extravascular lung water. Journal of Surgical Research 1990; 48:4549. DOI: 10.1016/0022-4804(90)90012-Q

4. Sirtoli V, Morcelles K, Gomez J and Bertemes-Filho $P$. Design and evaluation of an electrical bioimpedance device based on DIBS for myography during isotonic exercises. Journal of Low Power Electronics and Applications 2018; 8:50-73. DOI: 10.3390/jlpea8040050

5. Jakovljevic DG, Trenell MI and MacGowan GA. Bioimpedance and bioreactance methods for monitoring cardiac output. Best Practice \& Research Clinical Anaesthesiology 2014; 28:381-94. DOI: 10.1016/j.bpa.2014. 09.003

6. Hong S, Lee K, Ha U, Kim H, Lee $Y$, Kim $Y$ and Yoo HJ. A $4.9 \mathrm{~m} \Omega$-sensitivity mobile electrical impedance tomography IC for early breast-cancer detection system. IEEE Journal of Solid-State Circuits 2014; 50:245-57. DOI: 10.1109/JSSC.2014.2355835

7. Bertemes-Filho P. Tissue Characterisation using an Impedance Spectroscopy Probe. PhD thesis. Sheffield, $2002: 27-35$

8. Pease R. AN-1515 A Comprehensive Study of the Howland Current Pump. National Semiconductor 2013

9. Tucker AS, Fox RM and Sadleir RJ. Biocompatible, high precision, wideband, improved howland current source with lead-lag compensation. IEEE Transactions on Biomedical Circuits and Systems 2013; 7:63-70. DOI: 10.1109/TBCAS.2012.2199114

10. Storn R. On the usage of differential evolution for function optimization. en. Proceedings of North American Fuzzy Information Processing. Berkeley, CA, USA: IEEE, 1996 :519-23. DOI: 10.1109/NAFIPS.1996.534789

11. Jingqiao Zhang and Sanderson A. JADE: Adaptive Differential Evolution With Optional External Archive. en. IEEE Transactions on Evolutionary Computation 2009 Oct; 13:945-58. DOI: 10.1109/TEVC. 2009. 2014613

12. Morcelles KF, Sirtoli VG, Bertemes-Filho P and Vincence VC. Howland current source for high impedance load applications. Review of Scientific Instruments 2017 Nov; 88:114705. DOI: 10.1063/1.5005330 